\title{
A fast Monte Carlo algorithm for site or bond percolation
}

\author{
M. E. J. Newman ${ }^{1}$ and R. M. Ziff ${ }^{2}$ \\ ${ }^{1}$ Santa Fe Institute, 1399 Hyde Park Road, Santa Fe, NM 87501 \\ ${ }^{2}$ Michigan Center for Theoretical Physics and Department of Chemical Engineering, \\ University of Michigan, Ann Arbor, MI 48109
}

\begin{abstract}
We describe in detail a new and highly efficient algorithm for studying site or bond percolation on any lattice. The algorithm can measure an observable quantity in a percolation system for all values of the site or bond occupation probability from zero to one in an amount of time which scales linearly with the size of the system. We demonstrate our algorithm by using it to investigate a number of issues in percolation theory, including the position of the percolation transition for site percolation on the square lattice, the stretched exponential behavior of spanning probabilities away from the critical point, and the size of the giant component for site percolation on random graphs.
\end{abstract}

\section{INTRODUCTION}

Percolation [1] is certainly one of the best studied problems in statistical physics. Its statement is trivially simple: in site percolation every site on a specified lattice is independently either "occupied," with probability $p$, or not with probability $1-p$. The occupied sites form contiguous clusters which have some interesting properties. In particular, the system shows a continuous phase transition at a finite value of $p$ which, on a regular lattice, is characterized by the formation of a cluster large enough to span the entire system from one side to the other in the limit of infinite system size. We say such a system "percolates." As the phase transition is approached from small values of $p$, the average cluster size diverges in a way reminiscent of the divergence of fluctuations in the approach to a thermal continuous phase transition, and indeed one can define correlation functions and a correlation length in the obvious fashion for percolation models, and hence measure critical exponents for the transition.

One can also consider bond percolation in which the bonds of the lattice are occupied (or not) with probability $p$ (or $1-p$ ), and this system shows behavior qualitatively similar to though different in some details from site percolation.

Site and bond percolation have found a huge variety of uses in many fields. Percolation models appeared originally in studies of actual percolation in materials [1] percolation of fluids through rock for example [2 [4 - but have since been used in studies of many other systems, including granular materials [5.6], composite materials [7, polymers [8], concrete [9], aerogel and other porous media 10,11], and many others. Percolation also finds uses outside of physics, where it has been employed to model resistor networks [12], forest fires 13] and other ecological disturbances 114, epidemics 15, 16], robustness of the Internet and other networks 17, 18, biological evolution [19], and social influence [20], amongst other things. It is one of the simplest and best understood examples of a phase transition in any system, and yet there are many things about it that are still not known. For example, despite decades of effort, no exact solution of the site percolation problem yet exists on the simplest twodimensional lattice, the square lattice, and no exact results are known on any lattice in three dimensions or above. Because of these and many other gaps in our current understanding of percolation, numerical simulations have found wide use in the field.

Computer studies of percolation are simpler than most simulations in statistical physics, since no Markov process or other importance sampling mechanism is needed to generate states of the lattice with the correct probability distribution. We can generate states simply by occupying each site or bond on an initially empty lattice with independent probability $p$. Typically, we then want to find all contiguous clusters of occupied sites or bonds, which can be done using a simple depth- or breadth-first search. Once we have found all the clusters, we can easily calculate, for example, average cluster size, or look for a system spanning cluster. Both depth- and breadth-first searches take time $\mathrm{O}(M)$ to find all clusters, where $M$ is the number of bonds on the lattice. This is optimal, since one cannot check the status of $M$ individual bonds or adjacent pairs of sites in any less than $\mathrm{O}(M)$ operations [21]. On a regular lattice, for which $M=\frac{1}{2} z N$, where $z$ is the coordination number and $N$ is the number of sites, $\mathrm{O}(M)$ is also equivalent to $\mathrm{O}(N)$.

But now consider what happens if, as is often the case, we want to calculate the values of some observable quantity $Q$ (e.g., average cluster size) over a range of values of $p$. Now we have to perform repeated simulations at many different closely-spaced values of $p$ in the range of interest, which makes the calculation much slower. Furthermore, if we want a continuous curve of $Q(p)$ in the range of interest then in theory we have to measure $Q$ at an infinite number of values of $p$. More practically, we can measure it at a finite number of values and then interpolate between them, but this inevitably introduces some error into the results.

The latter problem can be solved easily enough. The trick [22]23] is to measure $Q$ for fixed numbers of occupied sites (or bonds) $n$ in the range of interest. Let us refer to the ensemble of states of a percolation system with exactly $n$ occupied sites or bonds as a "microcanon- 
ical percolation ensemble," the number $n$ playing the role of the energy in thermal statistical mechanics. The more normal case in which only the occupation probability $p$ is fixed is then the "canonical ensemble" for the problem. (If one imagines the occupied sites or bonds as representing particles instead, then the two ensembles would be "canonical" and "grand canonical," respectively. Some authors 24 have used this nomenclature.) Taking site percolation as an example, the probability of there being exactly $n$ occupied sites on the lattice for a canonical percolation ensemble is given by the binomial distribution:

$$
B(N, n, p)=\left(\begin{array}{l}
N \\
n
\end{array}\right) p^{n}(1-p)^{N-n} .
$$

(The same expression applies for bond percolation, but with $N$ replaced by $M$, the total number of bonds.) Thus, if we can measure our observable within the microcanonical ensemble for all values of $n$, giving a set of measurements $\left\{Q_{n}\right\}$, then the value in the canonical ensemble will be given by

$$
Q(p)=\sum_{n=0}^{N} B(N, n, p) Q_{n}=\sum_{n=0}^{N}\left(\begin{array}{c}
N \\
n
\end{array}\right) p^{n}(1-p)^{N-n} Q_{n} .
$$

Thus we need only measure $Q_{n}$ for all values of $n$, of which there are $N+1$, in order to find $Q(p)$ for all $p$. Since filling the lattice takes time $\mathrm{O}(N)$, and construction of clusters $\mathrm{O}(M)$, we take time $\mathrm{O}(M+N)$ for each value of $n$, and $\mathrm{O}\left(N^{2}+M N\right)$ for the entire calculation, or more simply $\mathrm{O}\left(N^{2}\right)$ on a regular lattice. Similar considerations apply for bond percolation.

In fact, one frequently does not want to know the value of $Q(p)$ over the entire range $0 \leq p \leq 1$, but only in the critical region, i.e., the region in which the correlation length $\xi$ is greater than the linear dimension $L$ of the system. If $\xi \sim\left|p-p_{c}\right|^{-\nu}$ close to $p_{c}$, where $\nu$ is a (constant) critical exponent, then the width of the critical region scales as $L^{-1 / \nu}$. Since the binomial distribution, Eq. (11), falls off very fast away from its maximum (approximately as a Gaussian with variance of order $N^{-1}$ ), it is safe also only to calculate $Q_{n}$ within a region scaling as $L^{-1 / \nu}$, and there are order $L^{d-1 / \nu}$ values of $N$ in such a region, where $d$ is the dimensionality of the lattice. Thus the calculation of $Q(p)$ in the critical region will take time of order $N L^{d-1 / \nu}=N^{2-1 / d \nu}$. In two dimensions, for example, $\nu$ is known to take the value $\frac{4}{3}$, and so we can calculate any observable quantity over the whole critical region in time $\mathrm{O}\left(N^{13 / 8}\right)$.

An alternative technique for calculating $Q(p)$ over a range of values of $p$ is to use a histogram method. (This should not be confused with the method of $\mathrm{Hu}$ 22, which is also referred to as a "histogram method," but which is different from the method described here. Our histogram method is the equivalent for a percolation model of the method of Ferrenberg and Swendsen [25] for thermal models.) Suppose that one performs a number $M$ of simulations of a percolation system, each one generating a single state $\mu$ of the system drawn from some probability distribution $P_{\mu}$. In each state we measure our observable of interest $Q$, giving a set of measurements $\left\{Q_{\mu}\right\}$. Then our estimate of the value of $Q(p)$ is given by

$$
Q(p)=\frac{\sum_{\mu} P_{\mu}^{-1} B\left(N, n_{\mu}, p\right) Q_{\mu}}{\sum_{\mu} P_{\mu}^{-1} B\left(N, n_{\mu}, p\right)},
$$

where $n_{\mu}$ is the number of occupied sites or bonds in state $\mu$. In normal (canonical) simulations of percolation $P_{\mu}$ is just equal to the binomial distribution $B\left(N, n_{\mu}, p\right)$, which then cancels out of Eq. (3) to give $Q(p)=\frac{1}{M} \sum_{\mu} Q_{\mu}$ in the usual fashion. In microcanonical simulations $P_{\mu}$ is uniform over values of $n$, and Eq. (3) is equivalent to Eq. (2). Suppose however that instead $P_{\mu}$ corresponds to the distribution of states produced by a canonical simulation at a different value $p_{0}$ of the site or bond occupation probability. In this case $P_{\mu}=B\left(N, n_{\mu}, p_{0}\right)$, and Eq. (3) becomes

$$
\begin{aligned}
Q(p) & =\frac{\sum_{\mu}\left[B\left(N, n_{\mu}, p\right) / B\left(N, n_{\mu}, p_{0}\right)\right] Q_{\mu}}{\sum_{\mu} B\left(N, n_{\mu}, p\right) / B\left(N, n_{\mu}, p_{0}\right)} \\
& =\frac{\sum_{\mu}\left[\left(\frac{1}{p_{0}}-1\right) /\left(\frac{1}{p}-1\right)\right]^{n_{\mu}} Q_{\mu}}{\sum_{\mu}\left[\left(\frac{1}{p_{0}}-1\right) /\left(\frac{1}{p}-1\right)\right]^{n_{\mu}}} .
\end{aligned}
$$

This equation tells us that if we perform a simulation or set of simulations at occupation probability $p_{0}$, and record the value(s) of $n_{\mu}$ and $Q_{\mu}$ for each state generated, we can use the results to estimate $Q(p)$ at any other value of $p$. In practice, the range over which we can reliably extrapolate away from $p_{0}$ using this method is limited by the range of values of $n$ sampled; if the values of $n$ sampled at $p_{0}$ make negligible contributions to the canonical ensemble of states at $p$, then extrapolation will give poor results. Since the values $n_{\mu}$ are drawn from a binomial distribution with width $\sqrt{N p_{0}\left(1-p_{0}\right)}$, the entire range of $n$ can be sampled with $\mathrm{O}\left(N^{1 / 2}\right)$ separate simulations at different values of $p_{0}$. Each simulation takes time $\mathrm{O}(N)$ to complete, and hence it takes time $\mathrm{O}\left(N^{3 / 2}\right)$ to calculate $Q(p)$ for all values of $p$. If we are only interested in the critical region, this time is reduced to $\mathrm{O}\left(N^{3 / 2-1 / d \nu}\right)$, which is $\mathrm{O}\left(N^{9 / 8}\right)$ in two dimensions. These times are considerably faster than those for the direct method described above of performing simulations for all values of $n$, but this speed is offset by the fact that the histogram method gives larger statistical errors on measured quantities than the direct method [26,27.

In this paper we describe a new algorithm which can perform the calculation of $Q(p)$ for all values of $p$ faster than any of the algorithms above, in time $\mathrm{O}(N)$. This is clearly far superior to the $\mathrm{O}\left(N^{2}\right)$ direct algorithm while having comparable accuracy in terms of statistical error. It is also substantially faster than the $\mathrm{O}\left(N^{3 / 2}\right)$ histogram method while having significantly better statistical accuracy. And even in the case where we only want to calculate $Q$ in the critical region, our algorithm is still faster 
doing all values of $n$, than previous algorithms doing only those in the critical region. (Because of the way it works, our algorithm cannot be used to calculate $Q_{n}$ only for the critical region; one is obliged to calculate $\mathrm{O}(N)$ values of $Q_{n}$.) A typical lattice size for percolation systems is about a million sites. On such a lattice our algorithm would be on the order of a million times faster than the simple $\mathrm{O}\left(N^{2}\right)$ method and a thousand times faster than the histogram method, and even just within the critical region it would still be around six times faster than the histogram method in two dimensions, while giving substantially better statistical accuracy.

The outline of this paper is as follows. In Section [I] we describe our algorithm. In Section III we give results from the application of the algorithm to three different example problems. In Section IV] we give our conclusions. We have reported some of the results given here previously in Ref. [28].

\section{THE ALGORITHM}

Our algorithm is based on a very simple idea. In the standard algorithms for percolation, one must create an entire new state of the lattice for every different value of $n$ one wants to investigate, and construct the clusters for that state. As various authors have pointed out, however [22,23,29, 30], if we want to generate states for each value of $n$ from zero up to some maximum value, then we can save ourselves some effort by noticing that a correct sample state with $n+1$ occupied sites or bonds is given by adding one extra randomly chosen site or bond to a correct sample state with $n$ sites or bonds. In other words, we can create an entire set of correct percolation states by adding sites or bonds one by one to the lattice, starting with an empty lattice.

Furthermore, the configuration of clusters on the lattice changes little when only a single site or bond is added, so that, with a little ingenuity, one can calculate the new clusters from the old with only a small amount of computational effort. This is the idea at the heart of our algorithm.

Let us consider the case of bond percolation first, for which our algorithm is slightly simpler. We start with a lattice in which all $M$ bonds are unoccupied, so that each of the $N$ sites is its own cluster with just one element. As bonds are added to the lattice, these clusters will be joined together into larger ones. The first step in our algorithm is to decide an order in which the bonds will be occupied. We wish to choose this order uniformly at random from all possible such orders, i.e., we wish to choose a random permutation of the bonds. One way of achieving this is the following:

1. Create a list of all the bonds in any convenient order. Positions in this list are numbered from 1 to $M$.

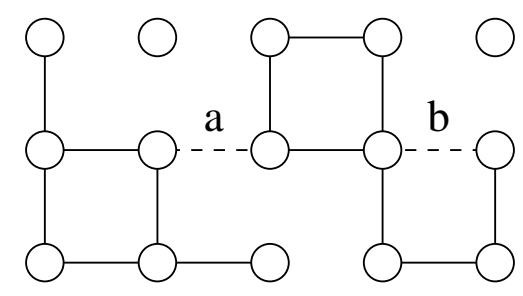

FIG. 1. Two examples of bonds (dotted lines) being added to bond-percolation configurations. (a) The added bond joins two clusters together. (b) The added bond does nothing, since the two joined sites were already members of the same cluster.

2. Set $i \leftarrow 1$.

3. Choose a number $j$ uniformly at random in the range $i \leq j \leq M$.

4. Exchange the bonds in positions $i$ and $j$. (If $i=j$ then nothing happens.)

5. Set $i \leftarrow i+1$.

6. Repeat from step 3 until $i=M$.

It is straightforward to convince oneself that all permutations of the bonds are generated by this procedure with equal probability, in time $\mathrm{O}(M)$, i.e., going linearly with the number of bonds on the lattice.

Having chosen an order for our bonds, we start to occupy them in that order. The first bond added will, clearly, join together two of our single-site clusters to form a cluster of two sites, as will, almost certainly, the second and third. However, not all bonds added will join together two clusters, since some bonds will join pairs of sites which are already part of the same cluster - see Fig. 1. Thus, in order to correctly keep track of the cluster configuration of the lattice, we must do two things for each bond added:

Find: when a bond is added to the lattice we must find which clusters the sites at either end belong to.

Union: if the two sites belong to different clusters, those clusters must be amalgamated into a single cluster; otherwise, if the two belong to the same cluster, we need do nothing.

Algorithms which achieve these steps are known as "union/find" algorithms [31,32. Union/find algorithms are widely used in data structures, in calculations on graphs and trees, and in compilers. They have been extensively studied in computer science, and we can make profitable use of a number of results from the computer science literature to implement our percolation algorithm simply and efficiently.

It is worth noting that measured values for lattice quantities such as, say, average cluster size, are not statistically independent in our method, since the configuration of the lattice changes at only one site from one step of the algorithm to the next. This contrasts with 


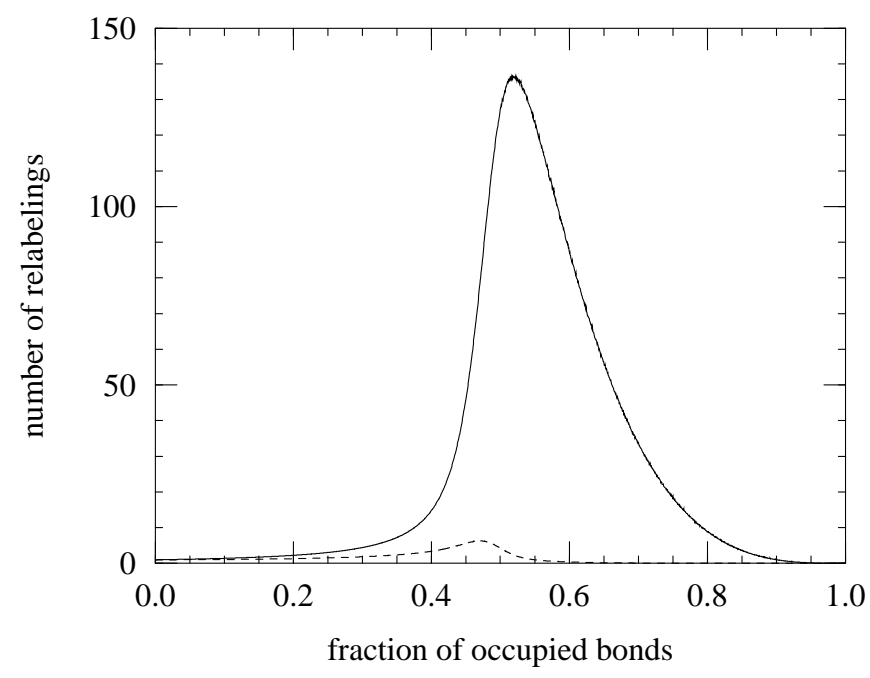

FIG. 2. Number of relabelings of sites performed, per bond added, as a function of fraction of occupied bonds, for the two algorithms described in Section II A, on a square lattice of $32 \times 32$ sites.

the standard algorithms, in which a complete new configuration is generated for every value of $n$ or $p$ investigated, and hence all data points are statistically independent. While this is in some respects a disadvantage of our method, it turns out that in most cases of interest it is not a problem, since almost always one is concerned only with statistical independence of the results from one run to another. This our algorithm clearly has, which means that an error estimate on the results made by calculating the variance over many runs will be a correct error estimate, even though the errors on the observed quantity for successive values of $n$ or $p$ will not be independent.

In the next two sections we apply a number of different and increasingly efficient union/find algorithms to the percolation problem, culminating with a beautifully simple and almost linear algorithm associated with the names of Michael Fischer and Robert Tarjan.

\section{A. Trivial (but possibly quite efficient) union/find algorithms}

Perhaps the simplest union/find algorithm which we can employ for our percolation problem is the following. We add a label to each site of our lattice - an integer for example - which tells us to which cluster that site belongs. Initially, all such labels are different (e.g., they could be set equal to their site label). Now the "find" portion of the algorithm is simple - we examine the labels of the sites at each end of an added bond to see if they are the same. If they are not, then the sites belong to different clusters, which must be amalgamated into one as a result of the addition of this bond. The amalgamation, the "union" portion of the algorithm, is

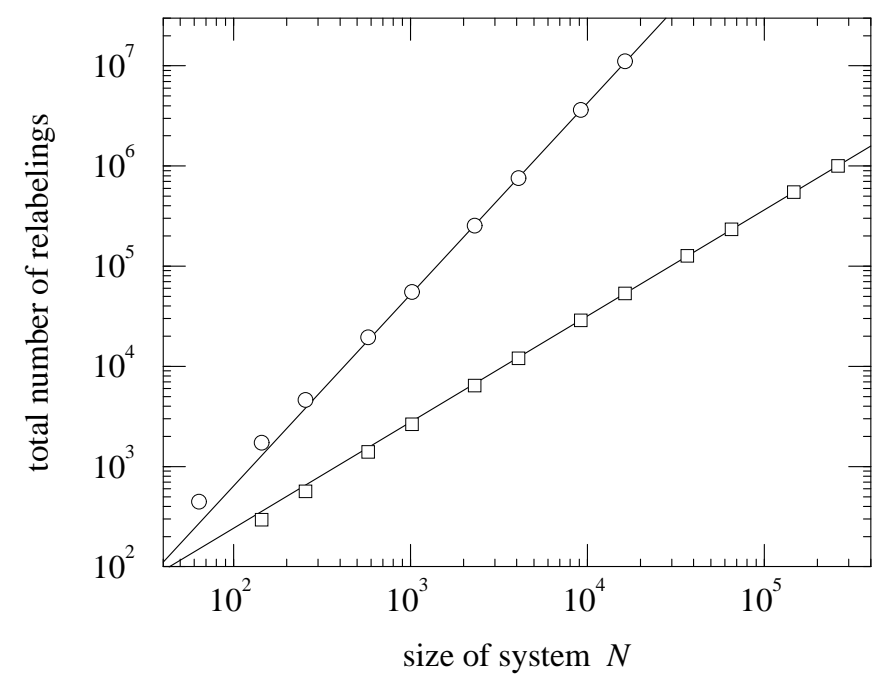

FIG. 3. Total number of relabelings of sites performed in a single run as a function of system size for the two algorithms (circles and squares) of Section IIA. Each data point is an average over 1000 runs; error bars are much smaller than the points themselves. The solid lines are straight-line fits to the rightmost five points in each case.

more involved. To amalgamate two clusters we have to choose one of them - in the simplest case we just choose one at random - and set the cluster labels of all sites in that cluster equal to the cluster label of the other cluster.

In the initial stages of the algorithm, when most bonds are unoccupied, this amalgamation step will be quick and simple; most bonds added will amalgamate clusters, but the clusters will be small and only a few sites will have to be relabeled. In the late stages of the algorithm, when most bonds are occupied, all or most sites will belong to the system-size percolating cluster, and hence cluster unions will rarely be needed, and again the algorithm is quick. Only in the intermediate regime, close to the percolation point, will any significant amount of work be required. In this region, there will in general be many large clusters, and much relabeling may have to be performed when two clusters are amalgamated. Thus, the algorithm displays a form of critical slowing down as it passes through the critical region. We illustrate this in Fig. 2 (solid line), where we show the number of site relabelings taking place, as a function of fraction of occupied bonds, for bond percolation on a square lattice of $32 \times 32$ sites. The results are averaged over a million runs of the algorithm, and show a clear peak in the number of relabelings in the region of the known percolation transition at bond occupation probability $p_{c}=\frac{1}{2}$.

In Fig. 3 (circles) we show the average total number of relabelings which are carried out during the entire run of the algorithm on square lattices of $N=L \times L$ sites as a function of $N$. Given that, as the number of relabelings becomes large, the time taken to relabel will be the dominant factor in the speed of this algorithm, this graph 
gives an indication of the expected scaling of run-time with system size. As we can see, the results lie approximately on a straight line on the logarithmic scales used in the plot and a fit to this line gives a run-time which scales as $N^{\alpha}$, with $\alpha=1.91 \pm 0.01$. This is slightly better than the $\mathrm{O}\left(N^{2}\right)$ behavior of the standard algorithms, and in practice can make a significant difference to running time on large lattices. With a little ingenuity however, we can do a lot better.

The algorithm's efficiency can be improved considerably by one minor modification: we maintain a separate list of the sizes of the clusters, indexed by their cluster label, and when two clusters are amalgamated, instead of relabeling one of them at random, we look up their sizes in this list and then relabel the smaller of the two. This "weighted union" strategy ensures that we carry out the minimum possible number of relabelings, and in particular avoids repeated relabelings of the large percolating cluster when we are above the percolation transition, which is a costly operation. The average number of relabelings performed in this algorithm as a function of number of occupied bonds is shown as the dotted line in Fig. 2, and it is clear that this change in the algorithm has saved us a great deal of work. In Fig. 3 (squares) we show the total number of relabelings as a function of system size, and again performance is clearly much better. The run-time of the algorithm now appears to scale as $N^{\alpha}$ with $\alpha=1.06 \pm 0.01$. In fact, we can prove that the worst-case running time goes as $N \log N$, a form which frequently manifests itself as an apparent power law with exponent slightly above 1 . To see this consider that with weighted union the size of the cluster to which a site belongs must at least double every time that site is relabeled. The maximum number of such doublings is limited by the size of the lattice to $\log _{2} N$, and hence the maximum number of relabelings of a site has the same limit. An upper bound on the total number of relabelings performed for all $N$ sites during the course of the algorithm is therefore $N \log _{2} N$. (A similar argument applied to the unweighted relabeling algorithm implies that $N^{2}$ is an upper bound on the running time of this algorithm. The measured exponent 1.91 indicates either that the experiments we have performed have not reached the asymptotic scaling regime, or else that the worst-case running time is not realized for the particular case of union of percolation clusters on a square lattice.)

An algorithm whose running time scales as $\mathrm{O}(N \log N)$ is a huge advance over the standard methods. However, it turns out that, by making use of some known techniques and results from computer science, we can make our algorithm better still while at the same time actually making it simpler. This delightful development is described in the next section.

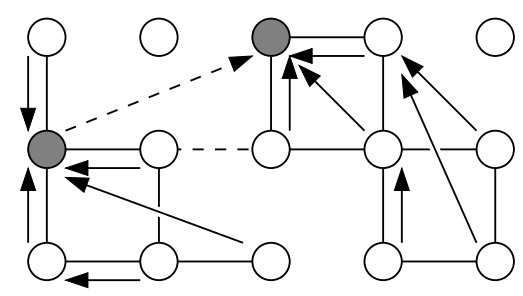

FIG. 4. An example of the tree structure described in the text, here representing two clusters. The shaded sites are the root sites and the arrows represent pointers. When a bond is added (dotted line in center) that joins sites which belong to different clusters (i.e., whose pointers lead to different root sites), the two clusters must be amalgamated by making one (left) a subtree of the other (right). This is achieved by adding a new pointer from the root of one tree to the root of the other.

\section{B. Tree-based union/find algorithms}

Almost all modern union/find algorithms make use of data trees to store the sets of objects (in this case clusters) which are to be searched. The idea of using a tree in this way seems to have been suggested first by Galler and Fischer [33. Each cluster is stored as a separate tree, with each vertex of the tree being a separate site in the cluster. Each cluster has a single "root" site, which is the root of the corresponding tree, and all other sites possess pointers either to that root site or to another site in the cluster, such that by following a succession of such pointers we can get from any site to the root. By traversing trees in this way, it is simple to ascertain whether two sites are members of the same cluster: if their pointers lead to the same root site then they are, otherwise they are not. This scheme is illustrated for the case of bond percolation on the square lattice in Fig. 5 .

The union operation is also simple for clusters stored as trees: two clusters can be amalgamated simply by adding a pointer from the root of one to any site in the other (Fig. (1). Normally, one chooses the new pointer to point to the root of the other cluster, since this reduces the average distance that will be have to be traversed across the tree to reach the root site from other sites.

There are many varieties of tree-based union/find algorithms, which differ from one another in the details of how the trees are updated as the algorithm progresses. However, the best known performance for any such algorithm is for a very simple one - the "weighted union/find with path compression" - which was first proposed by Fischer 31,32,34. Its description is brief:

Find: In the find part of the algorithm, trees are traversed to find their root sites. If two initial sites lead to the same root, then they belong to the same cluster. In addition, after the traversal is completed, all pointers along the path traversed are changed to point directly to the root of their tree. This is called "path compression." Path compression makes traversal faster the next time we perform a find operation on any of the same sites. 
Union: In the union part of the algorithm, two clusters are amalgamated by adding a pointer from the root of one to the root of the other, thus making one a subtree of the other. We do this in a "weighted" fashion as in Section II A, meaning that we always make the smaller of the two a subtree of the larger. In order to do this, we keep a record at the root site of each tree of the number of sites in the corresponding cluster. When two clusters are amalgamated the size of the new composite cluster is the sum of the sizes of the two from which it was formed.

Thus our complete percolation algorithm can be summarized as follows.

1. Initially all sites are clusters in their own right. Each is its own root site, and contains a record of its own size, which is 1 .

2. Bonds are occupied in random order on the lattice.

3. Each bond added joins together two sites. We follow pointers from each of these sites separately until we reach the root sites of the clusters to which they belong. Root sites are identified by the fact that they do not possess pointers to any other sites. Then we go back along the paths we followed through each tree and adjust all pointers along those paths to point directly to the corresponding root sites.

4. If the two root sites are the same site, we need do nothing further.

5. If the two root sites are different, we examine the cluster sizes stored in them, and add a pointer from the root of the smaller cluster to the root of the larger, thereby making the smaller tree a subtree of the larger one. If the two are the same size, we may choose whichever tree we like to be the subtree of the other. We also update the size of the larger cluster by adding the size of the smaller one to it.

These steps are repeated until all bonds on the lattice have been occupied. At each step during the run, the tree structures on the lattice correctly describe all of the clusters of joined sites, allowing us to evaluate observable quantities of interest. For example, if we are interested in the size of the largest cluster on the lattice as a function of the number of occupied bonds, we simply keep track of the largest cluster size we have seen during the course of the algorithm.

Although this algorithm may seem more involved than the relabeling algorithm of Section II A, it turns out that its implementation is actually simpler, for two reasons. First, there is no relabeling of sites, which eliminates the need for the code portion which tracks down all the sites belonging to a cluster and updates them. Second, the only difficult parts of the algorithm, the tree traversal and path compression, can it turns out be accomplished together by a single function which, by artful use of recursion, can be coded in only three lines (two in C). The implementation of the algorithm is discussed in detail in Section IID and in Appendix A.

\section{Performance of the algorithm}

Without either weighting or path compression each step of the algorithm above is known to take time linear in the tree size [34], while with either weighting or path compression alone it takes logarithmic time [34,35]. When both are used together, however, each step takes an amount of time which is very nearly constant, in a sense which we now explain.

The worst-case performance of the weighted union/find algorithm with path compression has been analyzed by Tarjan [35] in the general case in which one starts with $n$ individual single-element sets and performs all $n-1$ unions (each one reducing the number of sets by 1 from $n$ down to 1 ), and any number $m \geq n$ of intermediate finds. The finds are performed on any sequence of items, and the unions may be performed in any order. Our case is more restricted. We always perform exactly $2 M$ finds, and only certain unions and certain finds may be performed. For example, we only ever perform finds on pairs of sites which are adjacent on the lattice. And unions can only be performed on clusters which are adjacent to one another along at least one bond. However, it is clear that the worst-case performance of the algorithm in the most general case also provides a bound on the performance of the algorithm in any more restricted case, and hence Tarjan's result applies to our algorithm.

Tarjan's analysis is long and complicated. However, the end result is moderately straightforward. The union part of the algorithm clearly takes constant time. The find part takes time which scales as the number of steps taken to traverse the tree to its root. Tarjan showed that the average number of steps is bounded above by $k \alpha(m, n)$, where $k$ is an unknown constant and the function $\alpha(m, n)$ is the smallest integer value of $z$ greater than or equal to 1 , such that $A(z, 4\lceil m / n\rceil)>\log _{2} n$. The function $A(i, j)$ in this expression is a slight variant on Ackermann's function [36] defined by

$$
\begin{aligned}
& A(0, j)=2 j \quad \text { for all } j \\
& A(i, 0)=0 \quad \text { for } i \geq 1 \\
& A(i, 1)=2 \quad \text { for } i \geq 1 \\
& A(i, j)=A(i-1, A(i, j-1)) \quad \text { for } i \geq 1, j \geq 2 \text {. }
\end{aligned}
$$

This function is a monstrously quickly growing function of its arguments - faster than any multiple exponentialwhich means that $\alpha(m, n)$, which is roughly speaking the functional inverse of $A(i, j)$, is a very slowly growing function of its arguments. So slowly growing, in fact, that for all practical purposes it is a constant. In particular, note that $\alpha(m, n)$ is maximized by setting $m=n$, in which case if $\log _{2} n<A(z, 4)$ then $\alpha(m, n) \leq \alpha(n, n) \leq z$. Setting $z=3$ and making use of Eq. (5), we find that 


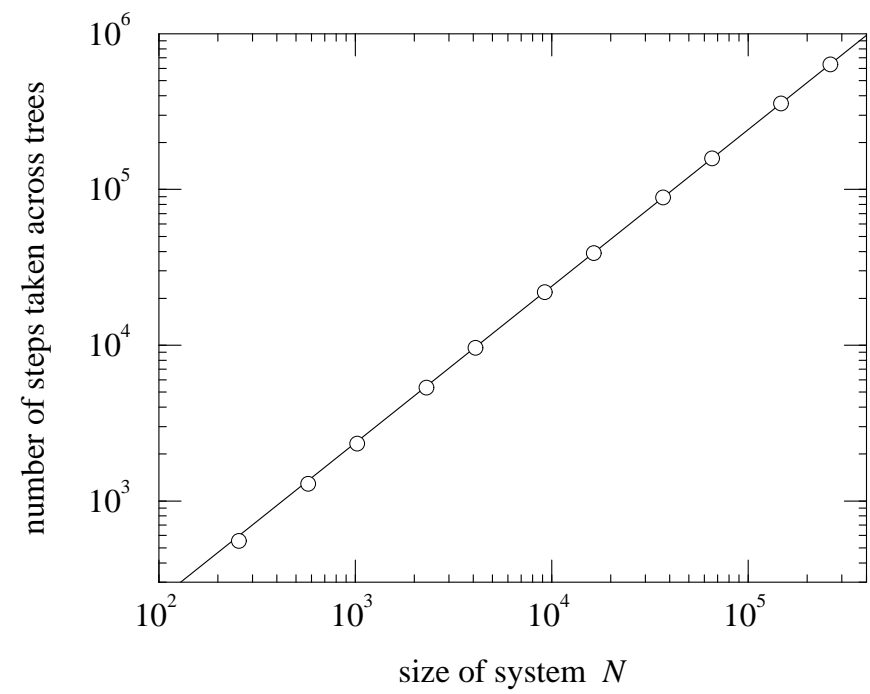

FIG. 5. Total number of steps taken through trees during a single run of the our algorithm as a function of system size. Each point is averaged over 1000 runs. Statistical errors are much smaller than the data points. The solid line is a straight-line fit to the last five points, and gives a slope of $1.006 \pm 0.011$.

$$
\left.A(3,4)=2^{2^{2 \cdots 2}}\right\} 65536 \text { twos. }
$$

This number is ludicrously large. For example, with only the first 5 out of the 65536 twos, we have

$$
2^{2^{2^{2^{2}}}}=2.0 \times 10^{19728}
$$

This number is far, far larger than the number of atoms in the known universe. Indeed, a generous estimate of the number of Planck volumes in the universe would put it at only around $10^{200}$. So it is entirely safe to say that $\log _{2} n$ will never exceed $A(3,4)$, bearing in mind that $n$ in our case is equal to $N$, the number of sites on the lattice. Hence $\alpha(m, n)<3$ for all practical values of $m$ and $n$ and certainly for all lattices which fit within our universe. Thus the average number of steps needed to traverse the tree is at least one and at most $3 k$, where $k$ is an unknown constant. The value of $k$ is found experimentally to be of order 1.

What does this mean for our percolation algorithm? It means that the time taken by both the union and find steps is $\mathrm{O}(1)$ in the system size, and hence that each bond added to the lattice takes time $\mathrm{O}(1)$. Thus it takes time $\mathrm{O}(M)$ to add all $M$ bonds, while maintaining an up-to-date list of the clusters at all times. (To be precise, the time to add all $M$ bonds is bounded above by $\mathrm{O}(M \alpha(2 M, N))$, where $\alpha(2 M, N)$ is not expected to exceed 3 this side of kingdom come.)

In Fig. 5 we show the actual total number of steps taken in traversing trees during the entire course of a run of the algorithm averaged over several runs for each

\begin{tabular}{lc}
\hline \hline algorithm & time in seconds \\
\hline depth-first search & 4500000 \\
unweighted relabeling & 16000 \\
weighted relabeling & 4.2 \\
tree-based algorithm & 2.9 \\
\hline \hline
\end{tabular}

TABLE I. Time in seconds for a single run of each of the algorithms discussed in this paper, for bond percolation on a square lattice of $1000 \times 1000$ sites.

data point, as a function of system size. A straight-line fit to the data shows that the algorithm runs in time proportional to $N^{\alpha}$, with $\alpha=1.006 \pm 0.011$. (In actual fact, if one measures the performance of the algorithm in real ("wallclock") time, it will on most computers (circa 2001) not be precisely linear in system size because of the increasing incidence of cache misses as $N$ becomes large. This however is a hardware failing, rather than a problem with the algorithm.)

We illustrate the comparative superiority of performance of our algorithm in Table If with actual timing results for it and the three other algorithms described in this paper, for square lattices of $1000 \times 1000$ sites, a typical size for numerical studies of percolation. All programs were compiled using the same compiler and run on the same computer. As the table shows, our algorithm, in addition to being the simplest to program, is also easily the fastest. In particular we find it to be about 2 million times faster than the simple depth-first search [37], and about $50 \%$ faster than the best of our relabeling algorithms, the weighted relabeling of Section IIA. For larger systems the difference will be greater still.

Site percolation can be handled by only a very slight modification of the algorithm. In this case, sites are occupied in random order on the lattice, and each site occupied is declared to be a new cluster of size 1 . Then one adds, one by one, all bonds between this site and the occupied sites, if any, adjacent to it, using the algorithm described above. The same performance arguments apply: generation of a permutation of the sites takes time $\mathrm{O}(N)$, and the adding of all the bonds takes time $\mathrm{O}(M)$, and hence the entire algorithm takes time $\mathrm{O}(M+N)$. On a regular lattice, for which $M=\frac{1}{2} z N$, where $z$ is the coordination number, this is equivalent to $\mathrm{O}(N)$.

It is worth also mentioning the memory requirements of our algorithm. In its simplest form, the algorithm requires two arrays of size $N$ for its operation; one is used to store the order in which sites will be occupied (for bond percolation this array is size $M$ ), and one to store the pointers and cluster sizes. The standard depth-first search also requires two arrays of size $N$ (a cluster label array and a stack), as does the weighted relabeling algorithm (a label array and a list of cluster sizes). Thus all the algorithms are competitive in terms of memory use. (The well-known Hoshen-Kopelman algorithm [38], 
which is a variation on depth-first search and runs in $\mathrm{O}\left(N^{2} \log N\right)$ time, has significantly lower memory requirements, of order $N^{1-1 / d}$, which makes it useful for studies of particularly large low-dimensional systems.)

\section{Implementation}

In Appendix A we give a complete computer program for our algorithm for site percolation on the square lattice written in $\mathrm{C}$. The program is also available for download on the Internet 39. As the reader will see, the implementation of the algorithm itself is quite simple, taking only a few lines of code. In this section, we make a few points about the algorithm's implementation, which may be helpful to those wishing to make use of it.

First, we note that the "find" operation in which a tree is traversed to find its root and the path compression can be conveniently performed together using a recursive function which in pseudocode would look like this:

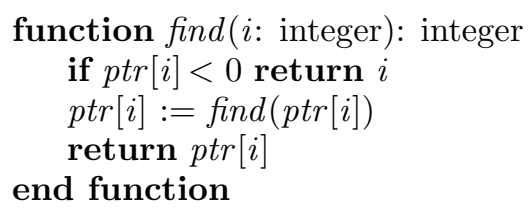

This function takes an integer argument, which is the label of a site, and returns the label of the root site of the cluster to which that site belongs. The pointers are stored in an array $p t r$, which is negative for all root nodes and contains the label of the site pointed to otherwise. A version of this function in $\mathrm{C}$ is included in Appendix A. A non-recursive implementation of the function is of course possible (as is the case with all uses of recursion), and we give two such implementations in the appendix for those who prefer this approach. However, both are somewhat more complicated than the recursive version, and with the benefit of a modern optimizing compiler are found to offer little speed advantage.

The program given in Appendix A measures the largest cluster size as a function of number of occupied sites. Above the percolation transition in the limit of large system size this quantity is equal to the size of the giant component, and it also has interesting scaling properties below the transition 40. However, there a many other quantities one might want to measure using our algorithm. Doing so usually involves keeping track of some additional variables as the algorithm runs. Here are three examples.

1. Average cluster size: In order to measure the average number of sites per cluster in site percolation it is sufficient to keep track of only the total number $c$ of clusters on the lattice. Then the average cluster size is given by $n / c$, where $n$ is the number of occupied sites. To keep track of $c$, we simply set it equal to zero initially, increase it by one every time a site is occupied, and decrease it

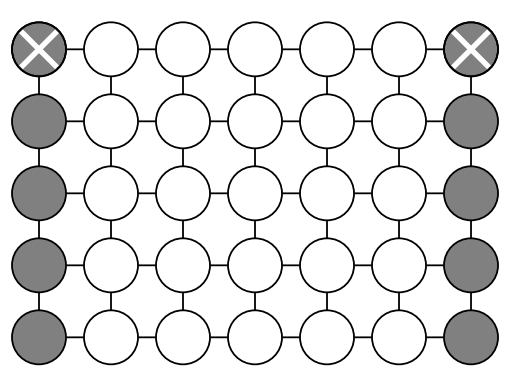

FIG. 6. Initial configuration of occupied (gray) and empty (white) sites for checking for the presence of a spanning cluster. In this example there are no periodic boundary conditions on the lattice. As the empty sites are filled up during the course of the run, the two sites marked $\times$ will have the same cluster root site if and only if there is a spanning cluster on the lattice.

by one every time we perform a union operation. Similarly, for averages weighted by cluster size one can keep track of higher moments of the cluster-size distribution.

2. Cluster spanning: In many calculations one would like to detect the onset of percolation in the system as sites or bonds are occupied. One way of doing this is to look for a cluster of occupied sites or bonds which spans the lattice from one side to the other. Taking the example of site percolation, one can test for such a cluster by starting the lattice in the configuration shown in Fig. 6 in which there are occupied sites along two edges of the lattice and no periodic boundary conditions. (Notice that the lattice is now not square.) Then one proceeds to occupy the remaining sites of the lattice one by one in our standard fashion. At any point, one can check for spanning simply by performing "find" operations on each of the two initial clusters, starting for example at the sites marked $\times$. If these two clusters have the same root site then spanning has occurred. Otherwise it has not.

3. Cluster wrapping: An alternative criterion for percolation is to use periodic boundary conditions and look for a cluster which wraps all the way around the lattice. This condition is somewhat more difficult to detect than simple spanning, but with a little ingenuity it can be done, and the extra effort is, for some purposes, worthwhile. An example is in the measurement of the position $p_{c}$ of the percolation transition. As discussed in Section III A, estimates of $p_{c}$ made using a cluster wrapping condition display significantly smaller finite-size errors than estimates made using cluster spanning on open systems.

A clever method for detecting cluster wrapping has been employed by Machta et al. [41] in simulations 


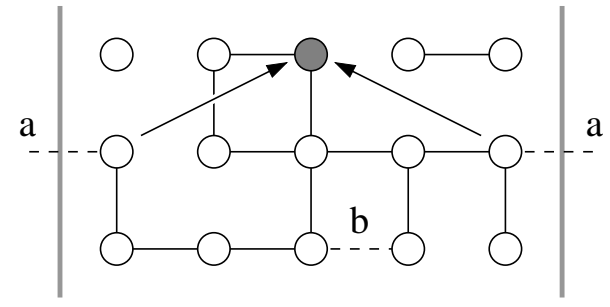

FIG. 7. Method for detecting cluster wrapping on periodic boundary conditions. When a bond is added (a) which joins together two sites which belong to the same cluster, it is possible, as here, that it causes the cluster to wrap around the lattice. To detect this, the displacements to the root site of the cluster (shaded) are calculated (arrows). If the difference between these displacements is not equal to a single lattice spacing, then wrapping has taken place. Conversely if the bond (b) where added, the displacements to the root site would differ by only a single lattice spacing, indicating that wrapping has not taken place.

of Potts models, and can be adapted to the case of percolation in a straightforward manner. We describe the method for bond percolation, although it is easily applied to site percolation also. We add to each site two integer variables giving the $x$ - and $y$ displacements from that site to the site's parent in the appropriate tree. When we traverse the tree, we sum these displacements along the path traversed to find the total displacement to the root site. (We also update all displacements along the path when we carry out the path compression.) When an added bond connects together two sites which are determined to belong to the same cluster, we compare the total displacements to the root site for those two sites. If these displacements differ by just one lattice spacing, then cluster wrapping has not occurred. If they differ by any other amount, it has. This method is illustrated in Fig. 7 .

It is worth noting also that, if one's object is only to detect the onset of percolation, then one can halt the algorithm as soon as percolation is detected. There is no need to fill in any more sites or bonds once the percolation point is reached. This typically saves about $50 \%$ on the run-time of the algorithm, the critical occupation probability $p_{c}$ being of the order of $\frac{1}{2}$. A further small performance improvement can often be achieved in this case by noting that it is no longer necessary to generate a complete permutation of the sites (bonds) before starting the algorithm. In many cases it is sufficient simply to choose sites (bonds) one by one at random as the algorithm progresses. Sometimes in doing this one will choose a site (bond) which is already occupied, in which case one must generate a new random one. The probability that this happens is equal to the fraction $p$ of occupied sites (bonds), and hence the average number of attempts we must make before we find an empty site is $1 /(1-p)$.
The total number of attempts made before we reach $p_{c}$ is therefore

$$
N \int_{0}^{p_{c}} \frac{\mathrm{d} p}{1-p}=-N \log \left(1-p_{c}\right),
$$

or $-M \log \left(1-p_{c}\right)$ for bond percolation. If $p_{c}=0.5$, for example, this means we will have to generate $N \log 2 \simeq$ $0.693 \mathrm{~N}$ random numbers during the course of the run, rather than the $N$ we would have had to generate to make a complete permutation of the sites. Thus it should be quicker not to generate the complete permutation. Only once $p_{c}$ becomes large enough that $-\log \left(1-p_{c}\right) \gtrsim 1$ does it start to become profitable to calculate the entire permutation, i.e., when $p_{c} \gtrsim 1-1 / \mathrm{e} \simeq 0.632$. If one were to use a random selection scheme for calculations over the whole range $0 \leq p \leq 1$, then the algorithm would take time

$$
N \int_{0}^{1-1 / N} \frac{\mathrm{d} p}{1-p}=N \log N
$$

to find and occupy all empty sites, which means overall operation would be $\mathrm{O}(N \log N)$ not $\mathrm{O}(N)$, so generating the permutation is crucial in this case to ensure that running time is $\mathrm{O}(N)$.

One further slightly tricky point in the implementation of our scheme is the performance of the convolution, Eq. (21), of the results of the algorithm with the binomial distribution. Since the number of sites or bonds on the lattice can easily be a million or more, direct evaluation of the binomial coefficients using factorials is not possible. And for high-precision studies, such as the calculations presented in Section III, a Gaussian approximation to the binomial is not sufficiently accurate. Instead, therefore, we recommend the following method of evaluation. The binomial distribution, Eq. (1), has its largest value for given $N$ and $p$ when $n=n_{\max }=p N$. We arbitrarily set this value to 1 . (We will fix it in a moment.) Now we calculate $B(N, n, p)$ iteratively for all other $n$ from

$$
B(N, n, p)= \begin{cases}B(N, n-1, p) \frac{N-n+1}{n} \frac{p}{1-p} & n>n_{\max } \\ B(N, n+1, p) \frac{n+1}{N-n} \frac{1-p}{p} & n<n_{\max } .\end{cases}
$$

Then we calculate the normalization coefficient $C=$ $\sum_{n} B(N, n, p)$ and divide all the $B(N, n, p)$ by it, to correctly normalize the distribution.

\section{APPLICATIONS}

In this section we consider a number of applications of our algorithm to open problems in percolation theory. Not all of the results given here are new-some have appeared previously in Refs. [18] and [28]. They are gathered here to give an idea of the types of problems for which our algorithm is an appropriate tool. 

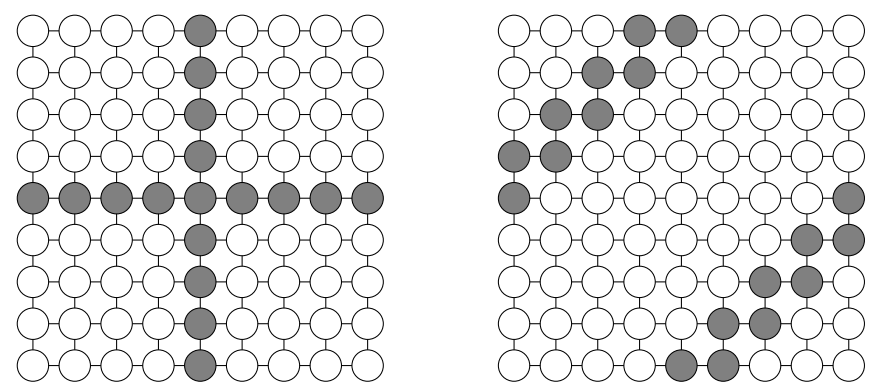

FIG. 8. Two topologically distinct ways in which a percolation cluster can wrap around both axes of a two-dimensional periodic lattice.

\section{A. Measurement of the position of the percolation transition}

Our algorithm is well suited to the measurement of critical properties of percolation systems, such as position of the percolation transition and critical exponents. Our first example application is the calculation of the position $p_{c}$ of the percolation threshold for site percolation on the square lattice, a quantity for which we currently have no exact result.

There are a large number of possible methods for determining the position of the percolation threshold numerically on a regular lattice 42 44, different methods being appropriate with different algorithms. As discussed in Ref. [28], our algorithm makes possible the use of a particularly attractive method based on lattice wrapping probabilities, which has substantially smaller finite-size scaling corrections than methods employed previously.

We define $R_{L}(p)$ to be the probability that for site occupation probability $p$ there exists a contiguous cluster of occupied sites which wraps completely around a square lattice of $L \times L$ sites with periodic boundary conditions. As in Section IID, cluster wrapping is here taken to be an estimator of the presence or absence of percolation on the infinite lattice. There are a variety of possible ways in which cluster wrapping can occur, giving rise to a variety of different definitions for $R_{L}$ :

- $R_{L}^{(h)}$ and $R_{L}^{(v)}$ are the probabilities that there exists a cluster which wraps around the boundary conditions in the horizontal and vertical directions respectively. Clearly for square systems these two are equal. In the rest of this paper we refer only to $R_{L}^{(h)}$.

- $R_{L}^{(e)}$ is the probability that there exists a cluster which wraps around either the horizontal or vertical directions, or both.

- $R_{L}^{(b)}$ is the probability that there exists a cluster which wraps around both horizontal and vertical directions. Notice that there are many topologically distinct ways in which this can occur. Two of them, the "cross" configuration and the "single spiral" configuration, are illustrated in Fig. \&. $R_{L}^{(b)}$ is defined to include both of these and all other possible ways of wrapping around both axes.

- $R_{L}^{(1)}$ is the probability that a cluster exists which wraps around one specified axis but not the other axis. As with $R_{L}^{(h)}$, it does not matter, for the square systems studied here, which axis we specify.

These four wrapping probabilities satisfy the equalities

$R_{L}^{(e)}=R_{L}^{(h)}+R_{L}^{(v)}-R_{L}^{(b)}=2 R_{L}^{(h)}-R_{L}^{(b)}$,

$R_{L}^{(1)}=R_{L}^{(h)}-R_{L}^{(b)}=R_{L}^{(e)}-R_{L}^{(h)}=\frac{1}{2}\left(R_{L}^{(e)}-R_{L}^{(b)}\right)$,

so that only two of them are independent. They also satisfy the inequalities $R_{L}^{(b)} \leq R_{L}^{(h)} \leq R_{L}^{(e)}$ and $R_{L}^{(1)} \leq$ $R_{L}^{(h)}$.

Each run of our algorithm on the $L \times L$ square system gives one estimate of each of these functions for the complete range of $p$. It is a crude estimate however: since an appropriate wrapping cluster either exists or doesn't for all values of $p$, the corresponding $R_{L}(p)$ in the microcanonical ensemble is simply a step function, except for $R_{L}^{(1)}$, which has two steps, one up and one down. All four functions can be calculated in the microcanonical ensemble by finding the numbers $n_{c}^{(h)}$ and $n_{c}^{(v)}$ of occupied sites at which clusters first appear which wrap horizontally and vertically. (This means that, as discussed in Section IID, the algorithm can be halted once wrapping in both directions has occurred, which for the square lattice gives a saving of about $40 \%$ in the amount of CPU time used.)

Our estimates of the four functions are improved by averaging over many runs of the algorithm, and the results are then convolved with the binomial distribution, Eq. (2), to give smooth curves of $R_{L}(p)$ in the canonical ensemble. (Alternatively, one can perform the convolution first and average over runs second; both are linear operations, so order is unimportant. However, the convolution is quite a lengthy computation, so it is sensible to choose the order that requires it to be performed only once.)

In Fig. 9 we show results from calculations of $R_{L}(p)$ using our algorithm for the four different definitions, for systems of a variety of sizes, in the vicinity of the percolation transition.

The reason for our interest in the wrapping probabilities is that their values can be calculated exactly. Exact expressions can be deduced from the work of Pinson 45] and written directly in terms of the Jacobi $\vartheta$-function $\vartheta_{3}(q)$ and the Dedekind $\eta$-function $\eta(q)$. Pinson calculated the probability, which he denoted $\pi(Z \times Z)$, of the occurrence on a square lattice of a wrapping cluster with the "cross" topology shown in the left panel of Fig. 8 . By duality, our probability $R_{\infty}^{(e)}\left(p_{c}\right)$ is just $1-\pi(Z \times Z)$, since if there is no wrapping around either axis, then 


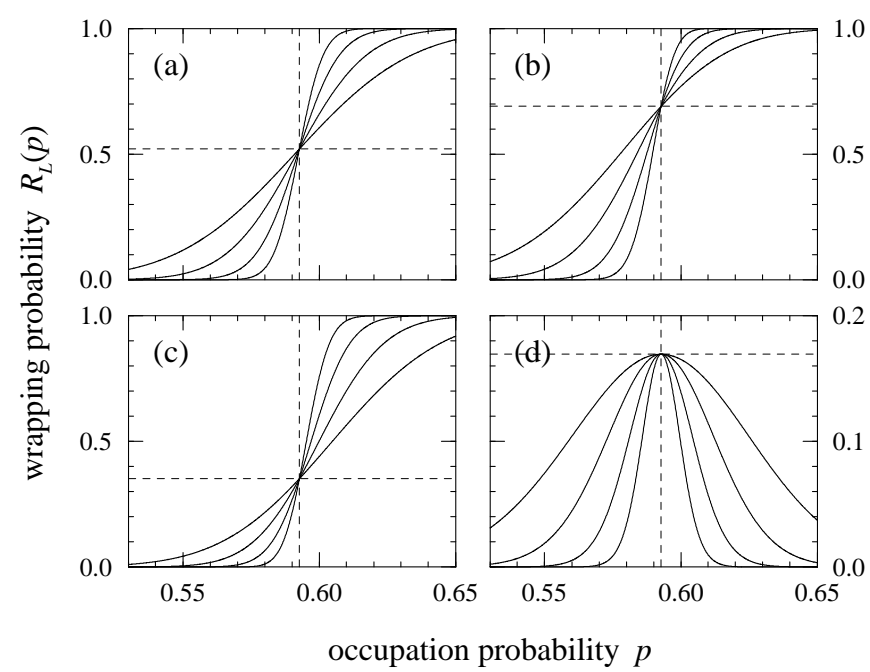

FIG. 9. Plots of the cluster wrapping probability functions $R_{L}(p)$ for $L=32,64,128$ and 256 in the region of the percolation transition for percolation (a) along a specified axis, (b) along either axis, (c) along both axes, and (d) along one axis but not the other. Note that (d) has a vertical scale different from the other frames. The dotted lines denote the expected values of $p_{c}$ and $R_{\infty}\left(p_{c}\right)$.

there must necessarily be a cross configuration on the dual lattice. This yields 46,47

$$
\begin{aligned}
& R_{\infty}^{(e)}\left(p_{c}\right)=1- \\
& \frac{\vartheta_{3}\left(\mathrm{e}^{-3 \pi / 8}\right) \vartheta_{3}\left(\mathrm{e}^{-8 \pi / 3}\right)-\vartheta_{3}\left(\mathrm{e}^{-3 \pi / 2}\right) \vartheta_{3}\left(\mathrm{e}^{-2 \pi / 3}\right)}{2\left[\eta\left(\mathrm{e}^{-2 \pi}\right)\right]^{2}} .
\end{aligned}
$$

The probability $R_{\infty}^{(1)}\left(p_{c}\right)$ for wrapping around exactly one axis is equal to the quantity denoted $\pi(1,0)$ by Pinson, which for a square lattice can be written

$$
R_{\infty}^{(1)}\left(p_{c}\right)=\frac{3 \vartheta_{3}\left(\mathrm{e}^{-6 \pi}\right)+\vartheta_{3}\left(\mathrm{e}^{-2 \pi / 3}\right)-4 \vartheta_{3}\left(\mathrm{e}^{-8 \pi / 3}\right)}{\sqrt{6}\left[\eta\left(\mathrm{e}^{-2 \pi}\right)\right]^{2}} .
$$

The remaining two probabilities $R_{\infty}^{(h)}$ and $R_{\infty}^{(b)}$ can now calculated from Eq. (12). To ten figures, the resulting values for all four are:

$$
\begin{aligned}
R_{\infty}^{(h)}\left(p_{c}\right)=0.521058290, & R_{\infty}^{(e)}\left(p_{c}\right)=0.690473725 \\
R_{\infty}^{(b)}\left(p_{c}\right)=0.351642855, & R_{\infty}^{(1)}\left(p_{c}\right)=0.169415435 .
\end{aligned}
$$

If we calculate the solution $p$ of the equation

$$
R_{L}(p)=R_{\infty}\left(p_{c}\right)
$$

we must have $p \rightarrow p_{c}$ as $L \rightarrow \infty$, and hence this solution is an estimator for $p_{c}$. Furthermore, it is a very good estimator, as we now demonstrate.

In Fig. 10, we show numerical results for the finite-size convergence of $R_{L}\left(p_{c}\right)$ to the $L=\infty$ values for both site

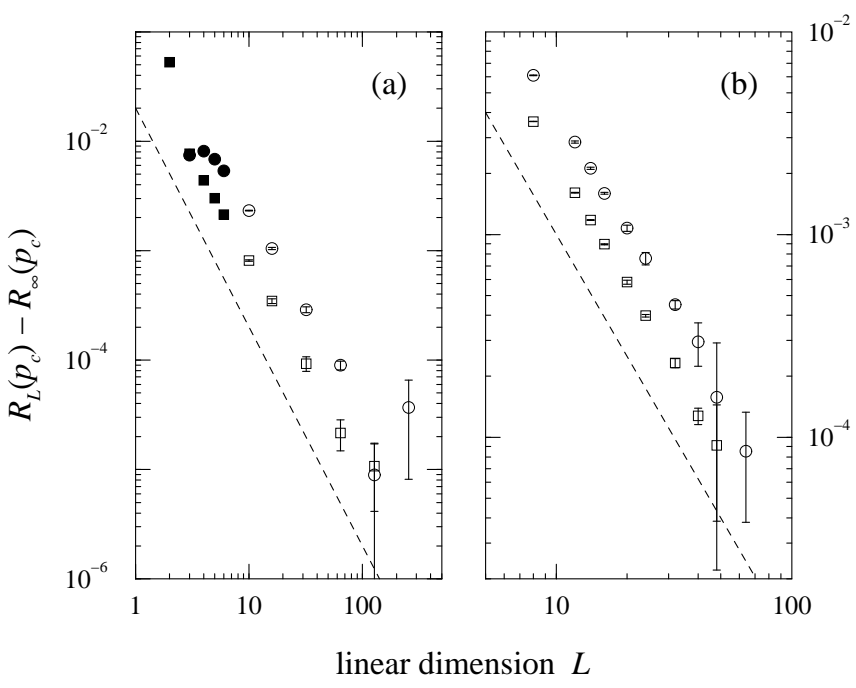

FIG. 10. Convergence with increasing system size of $R_{L}\left(p_{c}\right)$ to its known value at $L=\infty$ for $R_{L}^{(e)}$ (circles) and $R_{L}^{(1)}$ (squares) under (a) site percolation and (b) bond percolation. Filled symbols in panel (a) are exact enumeration results for system sizes $L=2 \ldots 6$. The dotted lines show the expected slope if, as we conjecture, $R_{L}\left(p_{c}\right)$ converges as $L^{-2}$ with increasing $L$. The data for $R_{L}^{(e)}$ in panel (b) have been displaced upwards by a factor of two for clarity.

and bond percolation. (For site percolation we used the current best-known value for $p_{c}$ from Ref. [28].) Note that the expected statistical error on $R_{L}\left(p_{c}\right)$ is known analytically to good accuracy, since each run of the algorithm gives an independent estimate of $R_{L}$ which is either 1 or 0 depending on whether or not wrapping has occurred in the appropriate fashion when a fraction $p_{c}$ of the sites are occupied. Thus our estimate of the mean of $R_{L}\left(p_{c}\right)$ over $n$ runs is drawn from a simple binomial distribution which has standard deviation

$$
\sigma_{R_{L}}=\sqrt{\frac{R_{L}\left(p_{c}\right)\left[1-R_{L}\left(p_{c}\right)\right]}{n}} .
$$

If we approximate $R_{L}\left(p_{c}\right)$ by the known value of $R_{\infty}\left(p_{c}\right)$, then we can evaluate this expression for any $n$.

As the figure shows, the finite-size corrections to $R_{L}$ decay approximately as $L^{-2}$ with increasing system size. For example, fits to the data for $R_{L}^{(1)}$ (for which our numerical results are cleanest), give slopes of $-1.95(17)$ (site percolation) and $-2.003(5)$ (bond percolation). On the basis of these results we conjecture that $R_{L}\left(p_{c}\right)$ converges to its $L=\infty$ value exactly as $L^{-2}$.

At the same time, the width of the critical region is decreasing as $L^{-1 / \nu}$, so that the gradient of $R_{L}(p)$ in the critical region goes as $L^{1 / \nu}$. Thus our estimate $p$ of the critical occupation probability from Eq. (16) converges to $p_{c}$ according to

$$
p-p_{c} \sim L^{-2-1 / \nu}=L^{-11 / 4},
$$


where the last equality makes use of the known value $\nu=\frac{4}{3}$ for percolation on the square lattice. This convergence is substantially better than that for any other known estimator of $p_{c}$. The best previously known convergence was $p-p_{c} \sim L^{-1-1 / \nu}$ for certain estimates based upon crossing probabilities in open systems, while many other estimates, including the renormalization-group estimate, converge as $p-p_{c} \sim L^{-1 / \nu}$ [44]. It implies that we should be able to derive accurate results for $p_{c}$ from simulations on quite small lattices. Indeed we expect that statistical error will overwhelm the finite size corrections at quite small system sizes, making larger lattices not only unnecessary, but also essentially worthless.

Statistical errors for the calculation are estimated in conventional fashion. From Eq. (17) we know that the error on the mean of $R_{L}(p)$ over $n$ runs of the algorithm goes as $n^{-1 / 2}$, independent of $L$, which implies that our estimate of $p_{c}$ carries an error $\sigma_{p_{c}}$ scaling according to $\sigma_{p_{c}} \sim n^{-1 / 2} L^{-1 / \nu}$. With each run taking time $\mathrm{O}(N)=\mathrm{O}\left(L^{d}\right)$, the total time $T \sim n L^{d}$ taken for the calculation is related to $\sigma_{p_{c}}$ according to

$$
\sigma_{p_{c}} \sim \frac{L^{d / 2-1 / \nu}}{\sqrt{T}}=\frac{L^{1 / 4}}{\sqrt{T}}
$$

where the last equality holds in two dimensions. Thus in order to make the statistical errors on systems of different size the same, we should spend an amount of time which scales as $T_{L} \sim L^{d-2 / \nu}$ on systems of size $L$, or $T_{L} \sim \sqrt{L}$ in two dimensions.

In Fig. 11 we show the results of a finite-size scaling calculation of this type for $p_{c}$. The four different definitions of $R_{L}$ give four (non-independent) estimates of $p_{c}$ : $0.59274621(13)$ for $R_{L}^{(h)}, 0.59274636(14)$ for $R_{L}^{(e)}$, $0.59274606(15)$ for $R_{L}^{(b)}$, and $0.59274629(20)$ for $R_{L}^{(1)}$. The first of these is the best, and is indeed the most accurate current estimate of $p_{c}$ for site percolation on the square lattice. This calculation involved the simulation of more than $7 \times 10^{9}$ separate samples, about half of which were for systems of size $128 \times 128$.

The wrapping probability $R_{L}^{(1)}$ is of particular interest, because one does not in fact need to know its value for the infinite system in order to use it to estimate $p_{c}$. Since this function is non-monotonic it may intercept its $L=\infty$ value at two different values of $p$, but its maximum must necessarily converge to $p_{c}$ at least as fast as either of these intercepts. And the position of the maximum can be determined without knowledge of the value of $R_{\infty}^{(1)}\left(p_{c}\right)$. In fact, in the calculation shown in Fig. 11, we used the maximum of $R_{L}^{(1)}$ to estimate $p_{c}$ and not the intercept, since in this case $R_{L}^{(1)}$ was strictly lower than $R_{\infty}^{(1)}$ for all $p$, so that there were no intercepts.

The criterion of a maximum in $R_{L}^{(1)}$ can be used to find the percolation threshold in other systems for which exact results for the wrapping probabilities are not known. As an example, we show in the inset of Fig. 11 a finitesize scaling calculation of $p_{c}$ for three-dimensional perco-

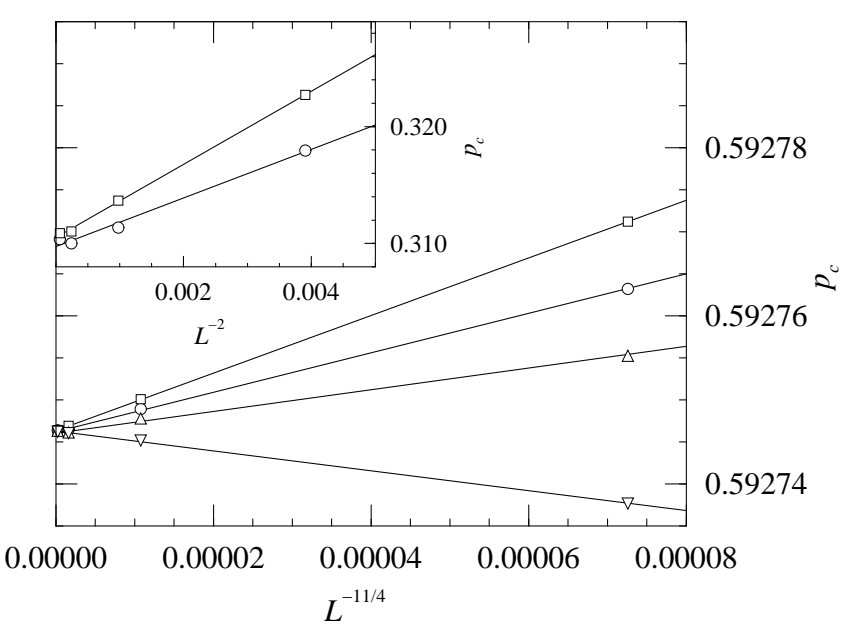

FIG. 11. Finite size scaling of estimate for $p_{c}$ on square lattices of $L \times L$ sites using measured probabilities of cluster wrapping along one axis (circles), either axis (squares), both axes (upward-pointing triangles), and one axis but not the other (downward-pointing triangles). Inset: results of a similar calculation for cubic lattices of $L \times L \times L$ sites using the probabilities of cluster wrapping along one axis but not the other two (circles), and two axes but not the other one (squares).

lation on the cubic lattice (with periodic boundary conditions in all directions) using this measure. Here there are two possible generalizations of our wrapping probability: $R_{L}^{(1)}(p)$ is the probability that wrapping occurs along one axis and not the other two, and $R_{L}^{(2)}(p)$ is the probability that wrapping occurs along two axes and not the third. We have calculated both in this case.

Although neither the exact value of $\nu$ nor the expected scaling of this estimate of $p_{c}$ is known for the threedimensional case, we can estimate $p_{c}$ by varying the scaling exponent until an approximately straight line is produced. This procedure reveals that our estimate of $p_{c}$ scales approximately as $L^{-2}$ in three dimensions, and we derive estimates of $p_{c}=0.3097(3)$ and $0.3105(2)$ for the position of the transition. Combining these results we estimate that $p_{c}=0.3101(10)$, which is in reasonable agreement with the best known result for this quantity of $0.3116080(4)$ [48, 49]. (Only a short run was performed to obtain our result; better results could presumably be derived with greater expenditure of CPU time.)

\section{B. Scaling of the wrapping probability functions}

It has been proposed 50,51 that below the percolation transition the probability of finding a cluster which spans the system, or wraps around it in the case of periodic boundary conditions, should scale according to

$$
R_{L}(p) \sim \exp (-L / \xi)
$$




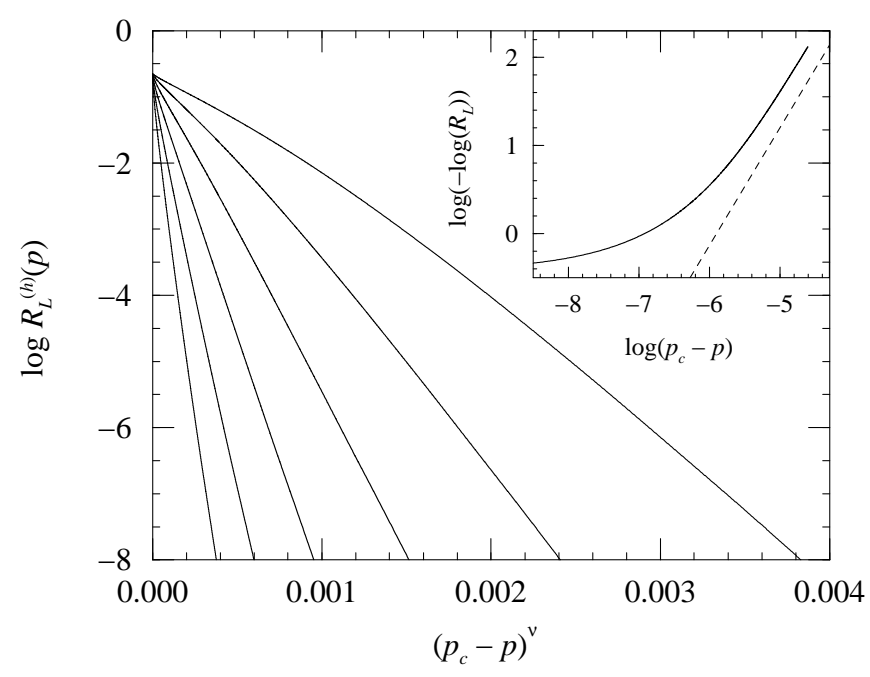

FIG. 12. Demonstration of the stretched exponential behavior, Eq. (21), in simulation results for $1024 \times 1024$ square systems. The results are averaged over 1000000 runs of the algorithm. The curves are for (top to bottom) $\nu=1.2,1.3$, $1.4,1.5,1.6$, and 1.7. Inset: the same data replotted logarithmically to show the stretched exponential behavior. The dotted line indicates the expected slope of $\frac{4}{3}$.

The probability of wrapping around the system is equal to the trace of the product of the transfer matrices for the $L$ rows of the system. With periodic boundary conditions, the transfer matrices are equal for all rows, and the wrapping probability is thus a simple sum of the $L$ th powers of the eigenvalues $\lambda_{i}$ of the individual transfer matrices: $R_{L}(p)=\sum_{i} \lambda_{i}^{L}$. For large $L$, this sum is dominated by the largest eigenvalue $\lambda_{0}$ and $R_{L}(p)=\lambda_{0}^{L}=\exp \left(L \log \lambda_{0}\right)$. Comparing with Eq. 200, we conclude that the leading constant in (20) must tend to unity as $L$ becomes large, and thus

$$
R_{L}(p)=\exp (-L / \xi)=\exp \left[-c L\left(p_{c}-p\right)^{\nu}\right],
$$

where $c$ is another constant. In other words, as a function of $p_{c}-p$, the wrapping probability should follow a stretched exponential with exponent $\nu$ and a leading constant of 1 . This contrasts with previous conjectures that $R_{L}(p)$ has Gaussian tails [1, 52, 53].

The behavior (21) is only seen when the correlation length is substantially smaller than the system dimension, but also greater than the lattice spacing, i.e., $1 \ll$ $\xi \ll L$. This means that in order to observe it clearly we need to perform simulations on reasonably large systems. In Fig. 12 we show results for site percolation on square lattices of $1024 \times 1024$ sites, with $\log R_{L}$ plotted against $\left(p_{c}-p\right)^{\nu}$ for various values of $\nu$, to look for straight-line behavior. Interpretation of the results is a little difficult, since one must discount curvature close to the origin where $\xi \gtrsim L$. However, the best straight line seems to occur in the region of $\nu=1.4 \pm 0.1$, in agree- ment with the expected $\nu=\frac{4}{3}$, while strongly ruling out the Gaussian behavior.

A better demonstration of this result is shown in the inset of the figure. Here we plot $\log \left(-\log \left(R_{L}\right)\right)$ as a function of $\log \left(p_{c}-p\right)$, which, since the leading constant in Eq. (21) is equal to unity, should give a straight line with slope $\frac{4}{3}$ in the regime where $1 \ll \xi \ll L$. This behavior is clearly visible in the figure. Note that this kind of plot is only valid for periodic boundary conditions, since the leading constant in Eq. (20) is not in general equal to 1 in other cases.

\section{Percolation on random graphs}

For our last example, we demonstrate the use of our algorithm on a system which is not built upon a regular lattice. The calculations of this section are instead performed on random graphs, i.e., collections of sites (or "vertices") with random bonds (or "edges") between them.

Percolation can be considered a simple model for the robustness of networks 17,18 . In a communications network, messages are routed from source to destination by passing them from one vertex to another through the network. In the simplest approximation, the network between a given source and destination is functional so long as a single path exists from source to destination, and non-functional otherwise. In real communications networks such as the Internet, a significant fraction of the vertices (routers in the case of the Internet) are nonfunctional at all times, and yet the majority of the network continues to function because there are many possible paths from each source to each destination, and it is rare that all such paths are simultaneously interrupted. The question therefore arises: what fraction of vertices must be non-functional before communications are substantially disrupted? This question may be rephrased as a site percolation problem in which occupied vertices represent functional routers and unoccupied vertices nonfunctional ones. So long as there is a giant component of connected occupied vertices (the equivalent of a percolating cluster on a regular lattice) then long-range communication will be possible on the network. Below the percolation transition, where the giant component disappears, the network will fragment into disconnected clusters. Thus the percolation transition represents the point at which the network as a whole becomes non-functional, and the size of the giant component, if there is one, represents the fraction of the network which can communicate effectively.

Both the position of the phase transition and the size of the giant component have been calculated exactly by Callaway et al. [18] for random graphs with arbitrary degree distributions. The degree $k$ of a vertex in a network is the number of other vertices to which it is connected. If $p_{k}$ is the probability that a vertex has degree $k$ and $q$ 


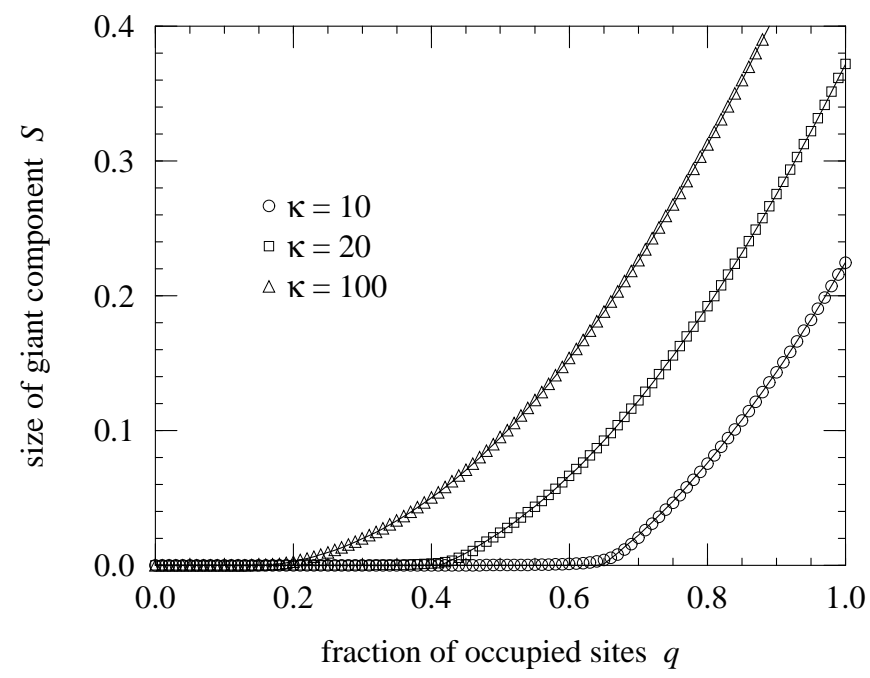

FIG. 13. Simulation results (points) for site percolation on random graphs with degree distribution given by Eq. (24), with $\tau=2.5$ and three different values of $\kappa$. The solid lines are the exact solution of Callaway et al. 18], Eq. (23), for the same parameter values.

is the occupation probability for vertices, then the percolation transition takes place at

$$
q_{c}=\frac{\sum_{k=0}^{\infty} k(k-1) p_{k}}{\sum_{k=0}^{\infty} k p_{k}},
$$

and the fraction $S$ of the graph filled by the giant component is the solution of

$$
S=q-q \sum_{k=0}^{\infty} p_{k} u^{k}, \quad u=1-q+q \frac{\sum_{k=0}^{\infty} k p_{k} u^{k-1}}{\sum_{k=0}^{\infty} k p_{k}} .
$$

For the Internet, the degree distribution has been found to be power-law in form [54], though in practice the power law must have some cutoff at finite $k$. Thus a reasonable form for the degree distribution is

$$
p_{k}=C k^{-\tau} \mathrm{e}^{-k / \kappa} \quad \text { for } k \geq 1 .
$$

The exponent $\tau$ is found to take values between 2.1 and 2.5 depending on the epoch in which the measurement was made and whether one looks at the network at the router level or at the coarser domain level. Vertices with degree zero are excluded from the graph since a vertex with degree zero is necessarily not a functional part of the network.

We can generate a random graph of $N$ vertices with this degree distribution in $\mathrm{O}(N)$ time using the prescription given in Ref. [55] and then use our percolation algorithm to calculate, for example, the size of the largest cluster for all values of $q$. In Fig. 13 we show the results of such a calculation for $N=1000000$ and $\tau=2.5$ for three different values of the cutoff parameter $\kappa$, along with the exact solution derived by numerical iteration of Eq. (23). As the figure shows, the two are in excellent agreement. The simulations for this figure took about an hour in total. We would expect a simulation performed using the standard depth-first search and giving results of similar accuracy to take about a million times as long, or about a century.

A number of authors 18,56, 57] have examined the resilience of networks to the selective removal of the vertices with highest degree. This scenario can also be simulated efficiently using our algorithm. The only modification necessary is that the vertices are now occupied in order of increasing degree, rather than in random order as in the previous case. We note however that the average time taken to sort the vertices in order of increasing degree scales as $\mathrm{O}(N \log N)$ when using standard sorting algorithms such as quicksort [31], and hence this calculation is dominated by the time to perform the sort for large $N$, making overall running time $\mathrm{O}(N \log N)$ rather than $\mathrm{O}(N)$.

\section{CONCLUSIONS}

We have described in detail a new algorithm for studying site or bond percolation on any lattice which can calculate the value of an observable quantity for all values of the site or bond occupation probability from zero to one in time which, for all practical purposes, scales linearly with lattice volume. We have presented a time complexity analysis demonstrating this scaling, empirical results showing the scaling and comparing running time to other algorithms for percolation problems, and a description of the details of implementation of the algorithm. A full working program is presented in the following appendix and is also available for download. We have given three example applications for our algorithm: the measurement of the position of the percolation transition for site percolation on a square lattice, for which we derive the most accurate result yet for this quantity; the confirmation of the expected $\frac{4}{3}$-power stretched exponential behavior in the tails of the wrapping probability functions for percolating clusters on the square lattice; and the calculation of the size of the giant component for site percolation on a random graph, which confirms the recently published exact solution for the same quantity.

\section{ACKNOWLEDGEMENTS}

The authors would like to thank Cris Moore, Barak Pearlmutter, and Dietrich Stauffer for helpful comments. This work was supported in part by the National Science Foundation and Intel Corporation. 


\section{APPENDIX A: PROGRAM}

In this appendix we give a complete program in $\mathrm{C}$ for our algorithm for site percolation on a square lattice of $N=L \times L$ sites with periodic boundary conditions. This program prints out the size of the largest cluster on the lattice as a function of number of occupied sites $n$ for values of $n$ from 1 to $N$. The entire program consists of 73 lines of code.

First we set up some constants and global variables:

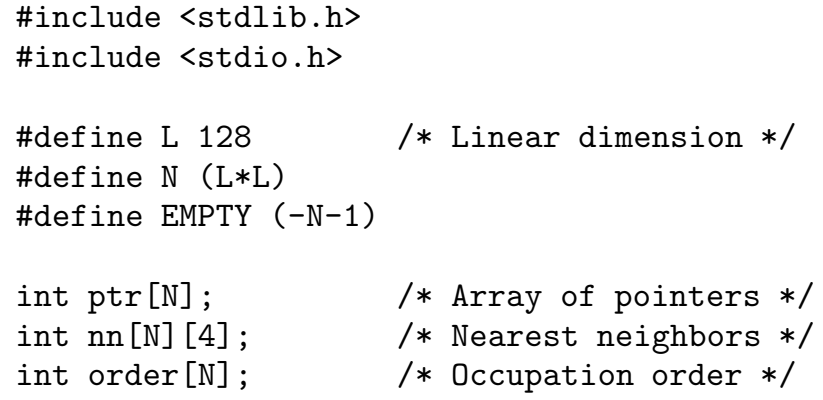

Sites are indexed with a single signed integer label for speed, taking values from 0 to $N-1$. Note that on computers which represent integers in 32 bits, this program can, for this reason, only be used for lattices of up to $2^{31} \simeq 2$ billion sites. While this is adequate for most purposes, longer labels will be needed if you wish to study larger lattices.

The array ptr [] serves triple duty: for non-root occupied sites it contains the label for the site's parent in the tree (the "pointer"); root sites are recognized by a negative value of $\operatorname{ptr}[]$, and that value is equal to minus the size of the cluster; for unoccupied sites ptr [] takes the value EMPTY.

Next we set up the array $n n[]$ [] which contains a list of the nearest neighbors of each site. Only this array need be changed in order for the program to work with a lattice of different topology.

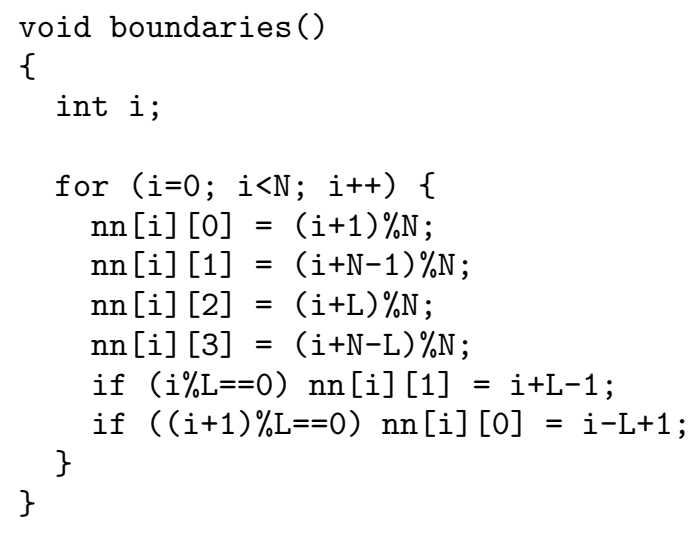

Now we generate the random order in which the sites will be occupied, by randomly permuting the integers from 0 to $N-1$ :

void permutation()

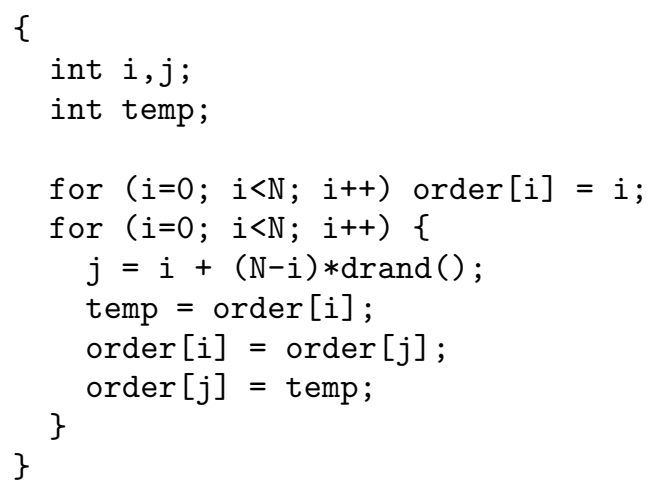

Here the function drand() generates a random double precision floating point number between 0 and 1. Many people will have such a function already to hand. For those who don't, a suitable one is supplied with Ref. [39].

We also define a function which performs the "find" operation, returning the label of the root site of a cluster, as well as path compression. The version we use is recursive, as described in Section IID:

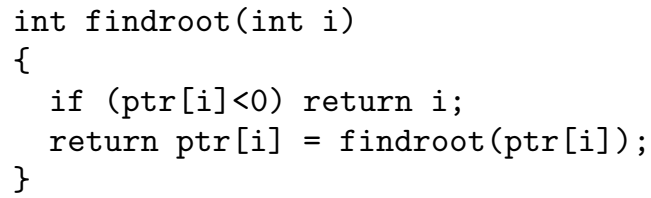

The code to perform the actual algorithm is quite brief. It works exactly as described in the text. Sites are occupied in the order specified by the array order []. The function findroot () is called to find the roots of each of the adjacent sites. If amalgamation is needed, it is performed in a weighted fashion, smaller clusters being added to larger (bearing in mind that the value of ptr [] for the root nodes is minus the size of the corresponding cluster).

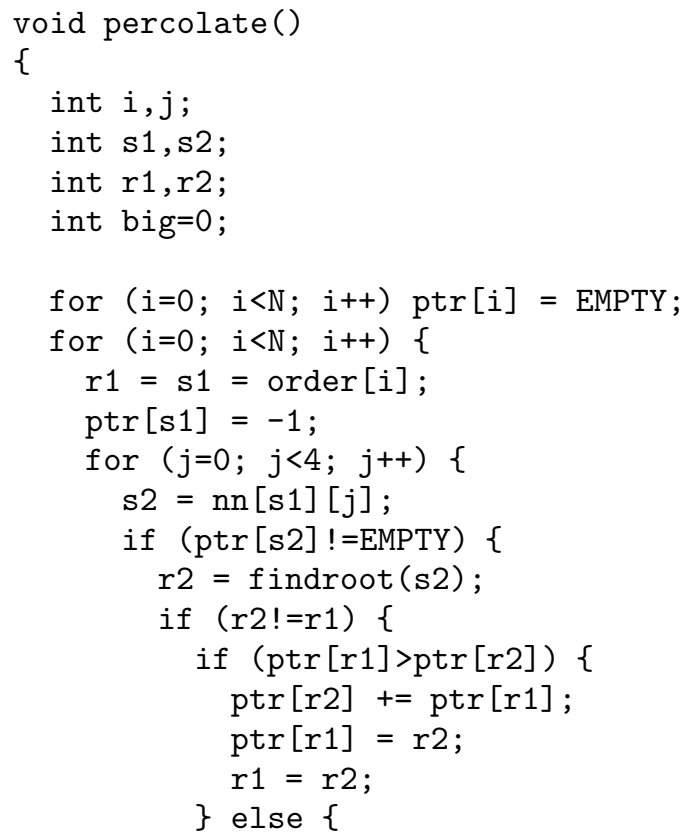




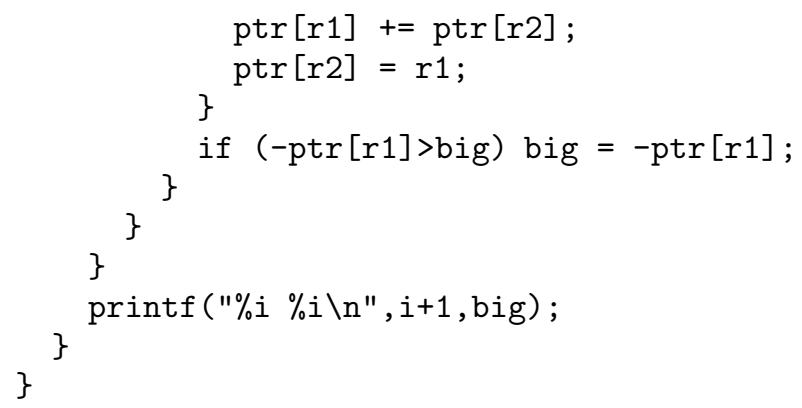

The main program is now simple:

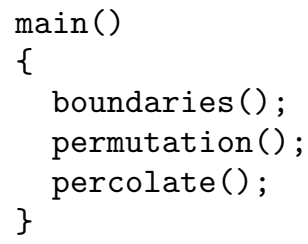

A complete working version of this program can also be downloaded from the Internet 39].

While our recursive implementation of the function findroot () is concise, some readers may find it unsatisfactory, either because they are using a compiler under which recursive code runs slowly, or because they want to translate the program into another language, such as Fortran 77, which does not support recursion. For their benefit we give here two alternative implementations of this function, neither of which makes use of recursion. The first of these is a straightforward implementation combining the find operation with path compression, as before, but using an explicit stack:

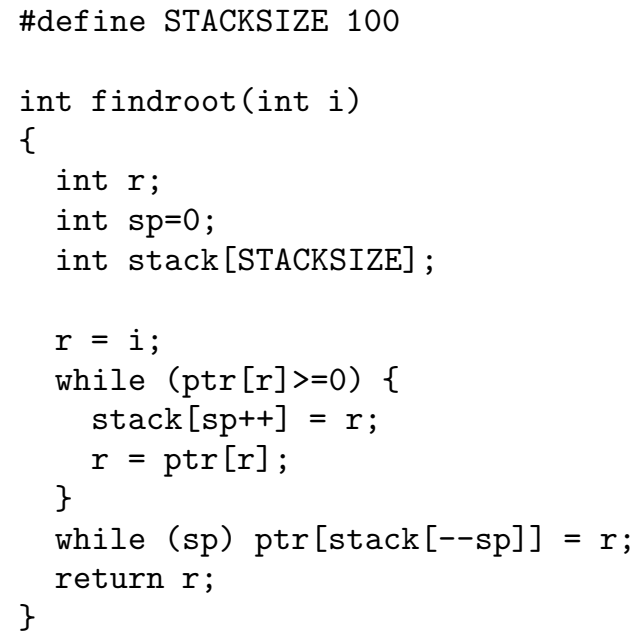

The stack used is small, having just 100 elements. This should be more than sufficient in almost all cases, since the average distance traversed across the tree is only about 3 .

A more elegant way to implement findroot() without recursion is to modify the union/find algorithm itself slightly. There is, it turns out, another union/find algorithm which runs in $\mathrm{O}(N)$ time. In this algorithm the union operation is as before, but the find operation now involves "path halving" instead of path compression. With path halving, each pointer along the path traversed is changed to point to its "grandparent" in the tree, which effectively halves the length of the path from a site to the root of the cluster each time findroot () is called. Tarjan [58] has shown that this find operation also runs asymptotically in very nearly constant time, giving an algorithm which runs in linear time overall. Here is a version of the function findroot () which implements path halving:

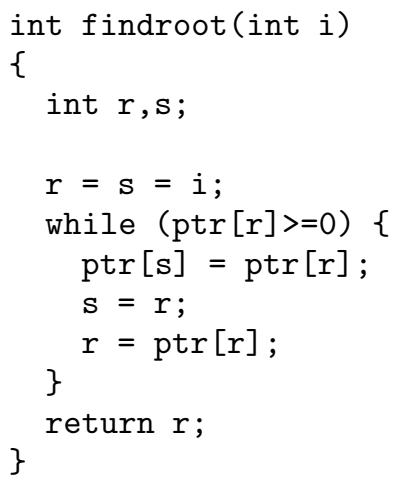

[1] D. Stauffer and A. Aharony, Introduction to Percolation Theory, 2nd edition, Taylor and Francis, London (1992).

[2] P. G. de Gennes and E. Guyon, J. de Mécanique 3, 403 (1978).

[3] R. G. Larson, L. E. Scriven, and H. T. Davis, Chem. Eng. Sci. 15, 57 (1981).

[4] M. Sahimi, J. Physique I 4, 1263 (1994).

[5] T. Odagaki and S. Toyofuku, J. Phys. CM 10, 6447 (1998).

[6] J. Tobochnik, Phys. Rev. E 60, 7137 (1999).

[7] S. de Bondt, L. Froyen, and A. Deruyttere, J. Mater. Sci. 27, 1983 (1992).

[8] A. Bunde, S. Havlin, and M. Porto, Phys. Rev. Lett. 74, 2714 (1995).

[9] D. P Bentz and E. J. Garboczi, Materials and Structures 25, 523 (1992).

[10] J. Machta, Phys. Rev. Lett. 66, 169 (1991).

[11] K. Moon and S. M. Girvin, Phys. Rev. Lett. 75, 1328 (1995).

[12] L. de Arcangelis, S. Redner, A. Coniglio, Phys. Rev. B 31, 4725 (1985).

[13] C. L. Henley, Phys. Rev. Lett. 71, 2741 (1993).

[14] K. A. With and T. O. Crist, Ecology 76, 2446 (1995).

[15] M. E. J. Newman and D. J. Watts, Phys. Rev. E 60, 7332 (1999).

[16] C. Moore and M. E. J. Newman, Phys. Rev. E 62, 7059 (2000).

[17] R. Cohen, K. Erez, D. ben-Avraham, and S. Havlin, Phys. Rev. Lett. 85, 4626 (2000). 
[18] D. S. Callaway, M. E. J. Newman, S. H. Strogatz, and D. J. Watts, Phys. Rev. Lett. 85, 5468 (2000).

[19] T. S. Ray and N. Jan, Phys. Rev. Lett. 72, 4045 (1994).

[20] S. Solomon, G. Weisbuch, L. de Arcangelis, N. Jan, and D. Stauffer, Physica A 277, 239 (2000).

[21] An algorithm does exist, for two dimensional systems only, which will generate the hull of the spanning cluster without populating the rest of the lattice. If one is interested only in this cluster, then this algorithm is faster. In particular, because the system-spanning hull in two dimensions is known to occupy a fraction of the lattice which scales as $N^{7 / 8}$ at criticality, the algorithm will run in time $\mathrm{O}\left(N^{7 / 8}\right)$ in the critical region. See R. M. Ziff, P. T. Cummings, and G. Stell, J. Phys. A 17, 3009 (1984).

[22] C.-K. Hu, Phys. Rev. B 46, 6592 (1992).

[23] H. Gould and J. Tobochnik, An Introduction to Computer Simulation Methods, 2nd edition, p. 444, AddisonWesley, Reading, MA (1996).

[24] L. N. Shchur and O A. Vasilyev, cond-mat/0005448.

[25] A. M. Ferrenberg and R. H. Swendsen, Phys. Rev. Lett. 61, 2635 (1988).

[26] A. M. Ferrenberg, D. P. Landau, and R. H. Swendsen, Phys. Rev. E 51, 5092 (1995).

[27] M. E. J. Newman and R. G. Palmer, J. Stat. Phys. 97, 1011 (1999).

[28] M. E. J. Newman and R. M. Ziff, Phys. Rev. Lett. 85, 4104 (2000).

[29] C. Moukarzel, Int. J. Mod. Phys. C 9, 887 (1998).

[30] J. E. de Freitas, L. S. Lucena, and S. Roux, Physica A 266, 81 (1999).

[31] R. Sedgewick, Algorithms, 2nd edition, Addison-Wesley, Reading, Mass. (1988).

[32] D. E. Knuth, The Art of Computer Programming, Vol. 1: Fundamental Algorithms, 3rd Edition, Addison-Wesley, Reading, Mass. (1997).

[33] B. A. Galler and M. J. Fischer, Comm. ACM 7, 301 (1964).

[34] M. J. Fischer, in Complexity of Computer Calculations, R. E. Miller and J. W. Thatcher (eds.), Plenum Press, New York (1972).

[35] R. E. Tarjan, J. ACM 22, 215 (1975).

[36] W. Ackermann, Math. Ann. 99, 118 (1928).

[37] We did not, as the reader may have guessed, actually run the depth-first search algorithm for 4.5 million seconds (about 2 months), since this would have been wasteful of computing resources. Instead, we ran the algorithm for 100 representative values of $n$, the number of occupied bonds, and scaled the resulting timing up to estimate the run-time for all 1000000 values.

[38] J. Hoshen and R. Kopelman, Phys. Rev. B 14, 3438 (1976).

[39] http://www. santafe.edu/ mark/percolation/

[40] M. Z. Bazant, Phys. Rev. E 62, 1660 (1999).

[41] J. Machta, Y. S. Choi, A. Lucke, T. Schweizer and L. M. Chayes, Phys. Rev. E 54, 1332 (1996).

[42] P. J. Reynolds, H. E. Stanley, and W. Klein, J. Phys. A 11, L199 (1978).

[43] P. J. Reynolds, H. E. Stanley, and W. Klein, Phys. Rev. $B$ 21, 1223 (1980).

[44] R. M. Ziff, Phys. Rev. Lett. 69, 2670 (1992).
[45] H. T. Pinson, J. Stat. Phys. 75, 1167 (1994).

[46] R. M. Ziff, C. D. Lorenz, and P. Kleban, Physica A 266, 17 (1999).

[47] Sometimes (for example, in the symbolic manipulation program Mathematica) the $\eta$-function is defined in complex argument form. If we were to use this definition, the $\eta$-functions in the denominators of Eqs. (13) and (14) would become $\eta(\mathrm{i})$.

[48] C. D. Lorenz and R. M. Ziff, J. Phys. A 31, 8147 (1998)

[49] H. G. Ballesteros, L. A. Fernández, V. Martín-Mayor, A. Muñoz Sudupe, G. Parisi, and J. J. Ruiz-Lorenzo, J. Phys. A 32, 1 (1999).

[50] L. Berlyand and J. Wehr, J. Phys. A 28, 7127 (1995).

[51] J.-P. Hovi and A. Aharony, Phys. Rev. E 53, 235 (1996).

[52] M. E. Levinshtein, B. I. Shklovskii, M. S. Shur, and A. L. Efros, Sov. Phys. JETP 42, 197 (1976).

[53] F. Wester, Int. J. Mod. Phys. C 11, 843 (2000).

[54] M. Faloutsos, P. Faloutsos, and C. Faloutsos, Comp. Comm. Rev. 29, 251 (1999).

[55] M. E. J. Newman, S. H. Strogatz, and D. J. Watts, cond-mat/0007235.

[56] R. Albert, H. Jeong, and A.-L. Barabási, Nature 406, 378 (2000).

[57] A. Broder, R. Kumar, F. Maghoul, P. Raghavan, S. Rajagopalan, R. Stata, A. Tomkins, and J. Wiener, Computer Networks 33, 309 (2000).

[58] R. E. Tarjan, Data Structures and Network Algorithms, Society for Industrial and Applied Mathematics, Philadelphia (1983). 